\title{
EVALUACIÓN DE OCRATOXINA A EN Theobroma cacao L. "cacao trinitario", POR CROMATOGRAFÍA LÍQUIDA DE ALTA EFICIENCIA (HPLC) Y ANÁLISIS MICOTOXIGÉNICO DURANTE EL PROCESO DE COSECHA, FERMENTADO, SECADO Y ALMACENADO
}

\author{
Norma Ramos $^{* a}$, Américo Castro ${ }^{a}$, Miguel Félix $^{\mathrm{a}}$, Félix Milla ${ }^{\mathrm{a}}$, Raúl Soria ${ }^{\mathrm{a}}$, \\ Mario Alcarraz ${ }^{\mathrm{a}}$, Donald Ramos ${ }^{\mathrm{b}}$, Fritz Choquesillo ${ }^{\mathrm{a}}$, Otilia Acha de la Cruz ${ }^{\mathrm{c}}$, \\ Jesús Blancas ${ }^{\mathrm{c}}$, Nadia Rodriguez ${ }^{\mathrm{c}}$, Diego Valdivieso ${ }^{\mathrm{a}}$
}

\begin{abstract}
RESUMEN
Se analizó la presencia de ocratoxina A(OTA), una micotoxina con propiedades carcinogénicas, en los granos de Theobroma cacao L. "cacao trinitario" procedente del centro de acopio de Pisana-Tingo María, de la Región San Martín, Perú. Las muestras fueron colectadas en cuatro etapas de producción: cosecha, fermentado, secado y almacenado. La OTA se extrajo utilizando acetonitrilo al $60 \%$ y purificándose mediante columnas de inmunoafinidad y posterior cuantificación por cromatografía líquida de alto rendimiento (HPLC). El porcentaje de recuperación del método utilizado se evaluó mediante el uso de muestras fortificadas con OTA evidenciando un porcentaje de recuperación mayor al $95 \%$. Los granos evaluados en las diferentes etapas de producción no manifestaron presencia de ocratoxina A y en el análisis micotoxigénico se evidenció la presencia de hongos del género Fusarium, Penicillium y levaduras.
\end{abstract}

Palabras clave: Ocratoxina A (OTA), cacao trinitario, columnas de inmunoafinidad, cromatografía líquida de alto rendimiento.

\section{EVALUATION OF OCHRATOXIN A IN Theobroma cacao L. "trinitario cacao", BY HIGH-EFFICIENCY LIQUID CHROMATOGRAPHY (HPLC) AND MICOTOXYGENIC ANALYSIS DURING THE PROCESS OF HARVESTING, FERMENTATION, DRYING AND STORAGE}

\footnotetext{
ABSTRACT

The presence of ochratoxin A(OTA), a mycotoxin with carcinogenic properties, was analyzed in the beans of Theobroma cacao L. "trinitario cacao" from the collection center of Pisana-

*a Facultad de Farmacia y Bioquímica, Universidad Nacional Mayor de San Marcos, Jr. Puno 1002. Jardín Botánico. Lima-Perú.nozjuli@gmail.com

b Facultad de Odontología, Universidad Nacional Mayor de San Marcos, Av. Germán Amézaga 375, Cercado de Lima. Lima-Perú.

c Facultad de Ciencias, Universidad Nacional de Ingeniería, Av. Túpac Amaru 210, Lima 25, Perú.
} 
Tingo Maria, of the San Martin Region, Peru. The samples were collected in four stages of production: harvesting, fermentation, drying and storage. The OTA was extracted using 60 $\%$ acetonitrile and purified by immunoaffinity columns and subsequent quantification by high performance liquid chromatography (HPLC). The recovery percentage of the method used was evaluated by using samples fortified with OTA, showing a recovery percentage greater than $95 \%$. The beans evaluated in the different stages of production did not show the presence of ochratoxin A and in the mycotoxigenic analysis the presence of fungi of the genus Fusarium, Penicillium and yeasts was evidenced.

Key words: Ochratoxin A (OTA), trinitarian cocoa, immunoaffinity columns, high performance liquid chromatography.

\section{INTRODUCCIÓN}

La ocratoxina A (OTA) es una toxina producida principalmente por hongos del género Aspergillus y Penicillium, la cual posee propiedades nefrotóxicas, inmunotóxicas, teratogénicas y carcinogénicas; y ataca diversos cultivos, entre ellos el cacao ${ }^{1}$.

El método de referencia para la cuantificación de micotoxinas es la Cromatografía Líquida de Alta Eficiencia (HPLC) con el sistema de detección de fluorescencia, debido a la sensibilidad para detectar concentraciones inferiores a 1 ppb (partes por billón) la cual ha demostrado ser eficiente en diversas muestras de estudio. Antes de cuantificar las muestras por HPLC, el contenido de OTA debe aislarse y extraerse. Si bien distintos métodos han sido utilizados para este fin, la extracción por cromatografía de inmunoafinidad es el método más difundido, ya que ha demostrado mayor eficacia y seguridad. El método se basa en la capacidad que tienen algunos anticuerpos para unirse de forma específica a las moléculas de OTA mediante una reacción antígeno-anticuerpo. De esta forma, los anticuerpos presentes en la fase estacionarían de las columnas utilizadas pueden retener el contenido de OTA, mientras dejan pasar los demás componentes de la muestra. Los hongos atacan diversos cultivos, existiendo variabilidad en las muestras de análisis, las cuales se comportan de manera diferentes frente a los solventes de extracción. Se debe evaluar el porcentaje de extracción del método a diferentes niveles. Esto se logra mediante el uso de muestras fortificadas, es decir; muestras que han sido contaminadas con una cantidad conocida del estándar analitico ${ }^{2,3}$.

Trabajos acerca de la presencia de OTA en cacao se han realizado en Nigeria, Costa de Marfil, Guinea, Camerún, Italia, Brasil, entre otros, concluyendo que los organismos generadores de toxina están presentes desde la cosecha, siendo la etapa de fermentación del cacao donde proliferan en mayor cantidad. De igual modo se ha demostrado que el secado del cacao y su almacenamiento en lugares secos con el cumplimento de normas técnicas y la implementación de buenas prácticas agrícolas-postcosecha, disminuyen en gran magnitud la presencia de OTA y otras micotoxinas ${ }^{4}$. En lo que respecta a la evaluación de los métodos, diversos autores han analizado los solventes de extracción utilizados, sus porcentajes de recuperación y demostrando que dichos porcentajes varían tanto por la matriz analizada 
como por el nivel de OTA evaluado. Entre los solventes evaluados para muestras de cacao se encuentran solución de bicarbonato de sodio, mezclas de metanol-agua, y mezclas de acetonitrilo-agua a diferentes concentraciones; obteniéndose diferentes porcentajes de recuperación dependiendo de la proporción de las mezclas ${ }^{2,5,6}$.

En el Perú, los estudios acerca de la presencia de ocratoxina A en cacao son escasos, destacando el realizado por Ramos et al. ${ }^{7}$, quien estudió su presencia en cacao piurano. Actualmente, el Perú presenta la necesidad de fijar un límite máximo permisible para OTA. Asimismo, no se cuenta con una norma de los niveles permitidos para el cacao y sus derivados; por ello, en este trabajo se realizó el análisis de la presencia de esta micotoxina en granos del cacao trinitario de la Región San Martín, la cual es una de las más importantes en volumen productivo de cacao $^{8}$.

En el presente trabajo se evaluó la presencia de ocratoxina A en muestras de granos de cacao y el análisis micotoxigénico durante las etapas de cosecha, fermentado, secado y almacenado del proceso de la producción del cacao. Este trabajo contribuye al estudio de la incidencia de ocratoxina A y la presencia de hongos generadores de micotoxinas en el cacao peruano, así como de los métodos utilizados para su determinación.

\section{PARTE EXPERIMENTAL}

El estudio se realizó en el Instituto de Investigación en Ciencias Farmacéuticas y Recursos Naturales "Juan de Dios Guevara” de la Facultad de Farmacia y Bioquímica de la Universidad Nacional Mayor de San Marcos y en el Laboratorio de Investigación y Certificaciones (LABICER) de la Facultad de Ciencias de la Universidad Nacional de Ingeniería.

\section{Reactivos}

Acetonitrilo de grado HPLC (J.T Baker), estándar de ocratoxina A (OTA) 99,18 \% (Merck), metanol $(\mathrm{MeOH})$ grado HPLC (Merck), ácido acético $\left(\mathrm{CH}_{3} \mathrm{COOH}\right)$ grado analítico $100 \%$ (Merck), Buffer fosfato salino en tabletas $\mathrm{pH}=7,40$ (Merck). Tween 20 para síntesis (Merck). El agua utilizada fue purificada en un equipo Elga Purelab CLASSIC UV.

\section{Equipos}

Cromatógrafo Líquido de Alto Rendimiento-SHIMADZU: desgasificador DGU-20A5R, automuestreador SIL-30AC, bomba cuaternaria LC-30AD, horno de columna: CTO-20AC, detector de fluorescencia RF-20Axs, control de sistema SBM-20A. Columna cromatográfica RP C18 de $250 \mathrm{~mm}$ x 4,6 mm ID x $5 \mu \mathrm{m}$ partícula (Restek). Molino de cuchillas, agitadores magnéticos de las marcas Thermo Scientific Cimarec y IKA C-MAG HS7. Balanza analítica digital SARTORIUS CPA225D, equipo ultrasonido WISD Laboratory Instruments, equipo de agua ultrapura ELGA PURELAB CLASSIC UV, columnas de inmunoafinidad para ocratoxina NEOCOLUMN. 


\section{Colecta de las muestras}

Los granos de Theobroma cacao L. "trinitario" fueron colectados del centro de acopio de Pisana-Tingo María, de la Región San Martín, Perú. Las actividades de muestreo se realizaron a una temperatura de $38{ }^{\circ} \mathrm{C}$ y $85 \%$ de humedad atmosférica durante el mes de junio del año 2017. Las muestras se colectaron en distintas etapas del proceso de la producción del cacao, las cuales consisten en: cosecha y apertura del fruto de cacao, fermentado en cajas durante seis días, secado al sol por seis días y, finalmente, almacenamiento para su posterior venta. Se seleccionaron cuatro tipos de muestra: cacao recién cosechado (M1), granos de cacao fermentados (M2), granos de cacao secados (M3) y granos de cacao almacenados en sacos (M4).

Las muestras M1 se colectaron de los frutos recién cosechados. Las demás muestras se colectaron en forma de granos.

\section{Pre-tratamiento de las muestras}

De las muestras M1, se extrajeron los granos y se secaron en estufa a $40{ }^{\circ} \mathrm{C}$ durante una semana; de igual forma se secaron las muestras M2 que habían sido colectadas húmedas. Las muestras M3 y M4 se secaron por un día en estufa a $40{ }^{\circ} \mathrm{C}$ para eliminar la humedad durante su almacenamiento. Las muestras se trataron en un mortero y luego en un molino de cuchillas. Una vez trituradas se homogenizaron y se conservaron a baja temperatura para su posterior análisis.

\section{Preparación de los estándares de ocratoxina A}

La solución madre del estándar de OTA se preparó mediante la disolución de 1 mg de estándar puro en $50 \mathrm{~mL}$ de Metanol (20 ppm). A partir de la solución madre se preparó la solución de trabajo (200 ppb) y a partir de esta solución se prepararon los diferentes estándares utilizados para la construcción de la curva de calibración, cuyas concentraciones variaron de 0,05 a 10 ppb. De la solución madre también se prepararon las soluciones utilizadas en las pruebas de recuperación del método utilizado.

\section{Extracción y purificación de la ocratoxina A en las muestras}

Este proceso se realizó por triplicado en cada uno de los cuatro tipos de muestra a analizar. De acuerdo al método descrito por Tafuri et al. ${ }^{5}$ para cacao, $25 \mathrm{~g}$ de muestra a analizar se re-suspendieron en $400 \mathrm{~mL}$ de acetonitrilo, se homogenizaron por 10 minutos con ayuda de un agitador magnético y se filtró con papel filtro Whatman $\mathrm{N}^{\circ} 42$; a $4 \mathrm{~mL}$ del filtrado se adhirieron a $44 \mathrm{~mL}$ de buffer PBS a pH: 7,4; los $48 \mathrm{~mL}$ de esta solución se purificaron en la columna de inmunoafinidad y se eluyeron a velocidad de 1-2 gotas por segundo con ayuda de una jeringa de $50 \mathrm{~mL}$. Las columnas se lavaron con $20 \mathrm{~mL}$ de buffer PBS pH=7.4-tween 20 al $0.01 \%$; y se eluyó el contenido de OTA con los siguientes solventes: $0,75 \mathrm{~mL}$ de metanol (Ac. Acético 2\%), 0,75 mL de metanol y 1,5 mL de agua ultrapura.

\section{Cuantificación por cromatografía líquida de alto rendimiento}

Los análisis se realizaron mediante cromatografía líquida de alto rendimiento con detección por fluorescencia siguiendo los parámetros recomendados por Tafuri et al ${ }^{5}$. 
La longitud de onda de excitación fue de $333 \mathrm{~nm}$ y la de emisión fue de $460 \mathrm{~nm}$. La temperatura del horno de la columna fue de $25{ }^{\circ} \mathrm{C}$. La elución fue a un flujo constante de $1 \mathrm{~mL} / \mathrm{min}$ y $\mathrm{CH}_{3} \mathrm{CN}_{\text {(ácido acético 1\%) }}-\mathrm{H}_{2} \mathrm{O}_{\text {(ácido acético } 1 \%)}(50: 50 \mathrm{v} / \mathrm{v})$ como el sistema de eluyentes inicial. El sistema inicial fue linealmente modificado a un $\mathrm{CH}_{3} \mathrm{CN} 100 \%$ en 20 minutos. Después de 3 minutos de permanecer constante, el sistema fue restablecido linealmente a su condición inicial 10 minutos, sumando un tiempo total de 33 minutos por análisis. Los eluyentes fueron preparados en el momento y filtrados $(0,45 \mu \mathrm{m})$ antes de su uso.

Para la lectura de los estándares se realizaron tres inyecciones y dos para el análisis de las muestras. Todas las muestras se filtraron a través de filtro de jeringa $(0,45 \mu \mathrm{m})$ antes de la inyección $(25 \mu \mathrm{L})$ a la columna cromatográfica.

La identificación de OTA se realizó utilizando el tiempo promedio de retención de las diluciones de trabajo. Este tiempo se obtuvo midiendo diez veces el estándar de trabajo a una concentración de $0,05 \mathrm{ppb}$, procedimiento que también fue útil para obtener el límite de detección (LDD). El tiempo de retención promedio fue de 8,245 minutos ( $\mathrm{RSD}=0,055 \%$ ) y el LDD fue de 0,100 ppb. En la figura 1 se muestra el cromatograma de una de las corridas del stock de trabajo de $0,05 \mathrm{ppb}$.

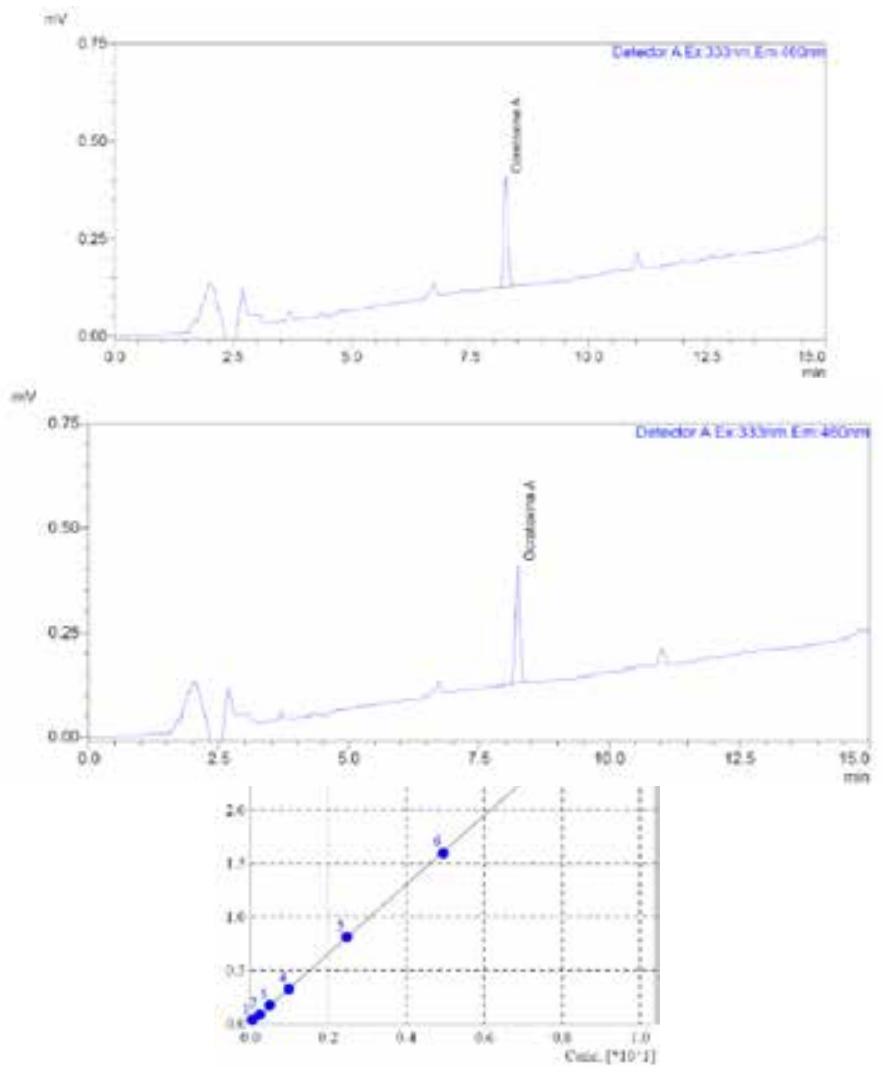

Figura 1. Curva de calibración utilizada para la cuantificación de la OTA. 


\section{Pruebas de recuperación del método utilizado}

El método utilizado fue evaluado para determinar su eficacia de recuperación; para esto, un blanco de granos frescos y secos, fue contaminado a tres niveles $(0,5 ; 7,5$ y $15 \mu \mathrm{g} / \mathrm{kg})$ con estándar de OTA. Las muestras contaminadas se trataron de igual manera que las demás.

\section{Análisis micotoxigénico}

Las semillas del cacao correspondientes a cada una de las etapas de procesamiento fueron sometidas a dos tipos de tratamiento para lograr aislar los posibles hongos asociados, el primer tratamiento consistió en colocar directamente las semillas sobre el agar APD y se procedió a incubar a $28^{\circ} \mathrm{C}$ durante $7-10$ días. En el segundo tratamiento, las semillas se sometieron a un proceso de lavado con solución salina estéril y tween 80 en agitación a $150 \mathrm{rpm}$ a temperatura ambiente durante 15 minutos, las soluciones obtenidas se diluyeron y se sembraron en agar de papa y dextrosa (APD) y se incubó a $28^{\circ} \mathrm{C}$ durante $7-10$ días. La identificación de los hongos se realizó mediante las estructuras reproductivas mostradas luego del microcultivo ${ }^{14,15}$.

\section{RESULTADOS Y DISCUSIÓN}

El método utilizado para el análisis de OTA fue evaluado en tres niveles de concentración $(0,5 ; 7,5$ y $15 \mu \mathrm{g} / \mathrm{kg})$. El nivel más bajo $(0,5 \mathrm{ug} / \mathrm{kg})$ fue elegido ya que es el límite máximo permitido sugerido por el gobierno de Italia $(0,5 \mu \mathrm{g} / \mathrm{kg})$, el cual es el único país europeo que actualmente establece un límite máximo para la OTA en cacao.

En la tabla 1 se muestran los resultados de las pruebas de recuperación con sus respectivas desviaciones estándar tal cual fueron calculadas utilizando el Excel Office 2016.

Tabla 1. Resultados de las pruebas de recuperación.

\begin{tabular}{cc}
\hline Nivel de contaminación $(\mathrm{ug} / \mathrm{Kg})$ & Porcentaje de recuperación (\%) \\
\hline 0,5 & $95,57 \pm 0,51$ \\
7,5 & $98,21 \pm 0,48$ \\
15 & $98,73 \pm 0,33$ \\
\hline
\end{tabular}

El cromatograma de la prueba de recuperación a un nivel de contaminación de 7,5ug/ $/ \mathrm{kg}$ se presenta en la figura 3. 


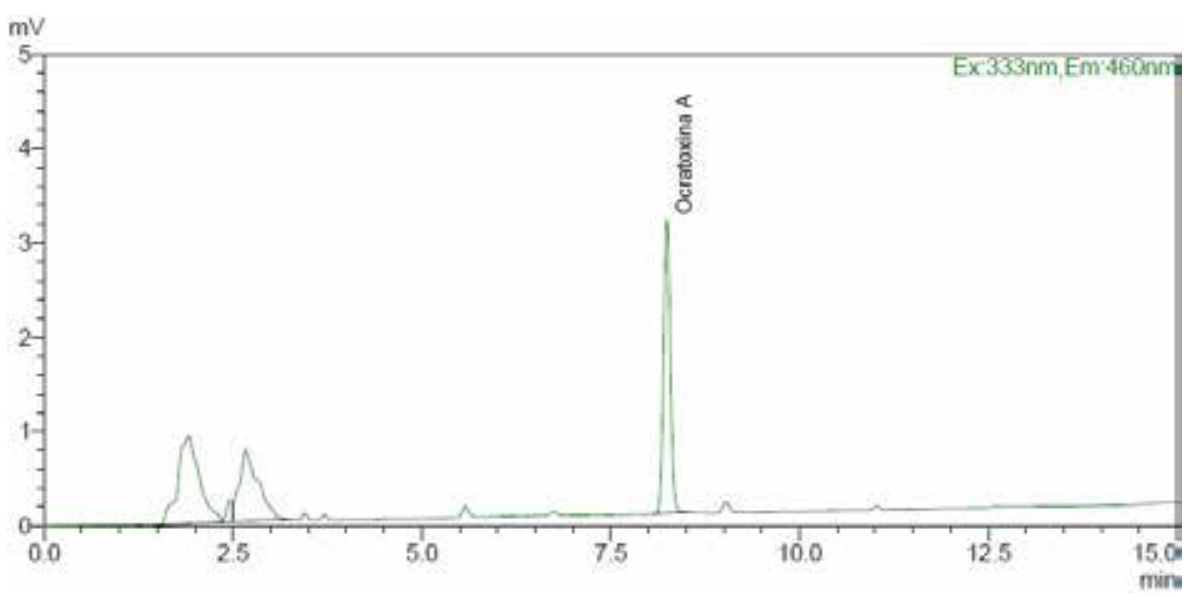

Figura 2. Cromatograma de la prueba de recuperación a un nivel de 7,5 ug/ $\mathrm{kg}$.

Los resultados demuestran que el método utilizado es eficaz para el análisis de OTA en muestras de granos de cacao. La eficacia de un método de extracción depende principalmente del solvente de extracción, el tipo de muestra a analizar, la recuperación de OTA de la columna de inmunoafinidad y la concentración de OTA en la muestra ${ }^{6}$. Debido a esto, los fabricantes de columnas de inmunoafinidad recomiendan diferentes métodos de extracción para diferentes tipos de muestras. El fabricante de las columnas utilizadas (Neogen - Neocolumn) no posee un método en especial para muestras de cacao, sin embargo, otros fabricantes tienen métodos especializados para este tipo de muestras. De ellos, resalta el fabricante R-Biophram - Ochraprep quien posee un método para cacao en polvo y otro para cascarilla de cacao. El primero utiliza bicarbonato de sodio $1 \%$ y el segundo acetonitrilo $60 \%$. Se decidió utilizar el segundo método ya que en el caso de granos de cacao la OTA suele encontrarse en la cáscara y muy rara vez la atraviesa. El uso de acetonitrilo $60 \%$ es también recomendado para el análisis de cereales, los cuales son matrices muy similares a los de la cascarilla de cacao $^{9}$. Adicionalmente, el acetonitrilo $60 \%$ es un método recomendado para diversos tipos de matrices ya que permite la precipitación de proteínas y posee una polaridad intermedia, adecuada para solubilizar las moléculas de OTA (molécula de polaridad media) ${ }^{10}$.

El hecho de que el método utilizado haya mostrado un porcentaje de recuperación mayor al $95 \%$ para las tres muestras analizadas, corrobora su eficacia para este tipo de muestra. Los métodos de recuperación suelen aumentar su eficacia conforme la concentración de OTA aumenta, tal cual se muestra en el presente trabajo. Esta eficacia se mantiene hasta una concentración máxima en la cual la columna de inmunoafinidad se sature de OTA. Entre los factores que disminuyen la eficacia de un método de extracción se encuentran: pérdida de muestra durante la ejecución del método, alta velocidad de goteo del solvente de recuperación a la hora de recuperar la OTA de la columna de inmunoafinidad y la eficacia del solvente de recuperación5. En este caso, el uso de metanol acidificado demostró ser un excelente solvente de recuperación, y la velocidad de dos gotas por segundo demostró ser adecuada. 
El metanol acidificado es bastante adecuado para este fin, desnaturalizando los anticuerpos (estructuras proteicas) de las columnas de inmunoafinidad, además rompe los enlaces entre la OTA y dichos anticuerpos.

En el presente estudio se analizó el contenido de OTA de muestras de granos de cacao en cuatro etapas de la producción: cosecha, fermentado, secado y almacenado. Los resultados de los análisis se muestran en la tabla 2.

Tabla 2. Contenido de OTA en las cuatro etapas analizadas.

\begin{tabular}{cc}
\hline Etapa & Contenido de OTA $(\mu \mathrm{g} / \mathrm{Kg})$ \\
\hline Cosecha & $<\mathrm{N} . \mathrm{D}$ \\
Fermentado & $<\mathrm{N} . \mathrm{D}$ \\
Secado & $<\mathrm{N} . \mathrm{D}$ \\
Almacenado & $<\mathrm{N} . \mathrm{D}$ \\
\hline
\end{tabular}

N. $\mathrm{D}=$ No detectable

Todas las muestras estudiadas muestran niveles de OTA menores al límite de detección. Estos resultados contrastan con los encontrados previamente por Ramos et al.7, los cuales mostraron presencia de OTA en la etapa de fermentado. El cromatograma de una de las muestras se presenta en la figura 4.

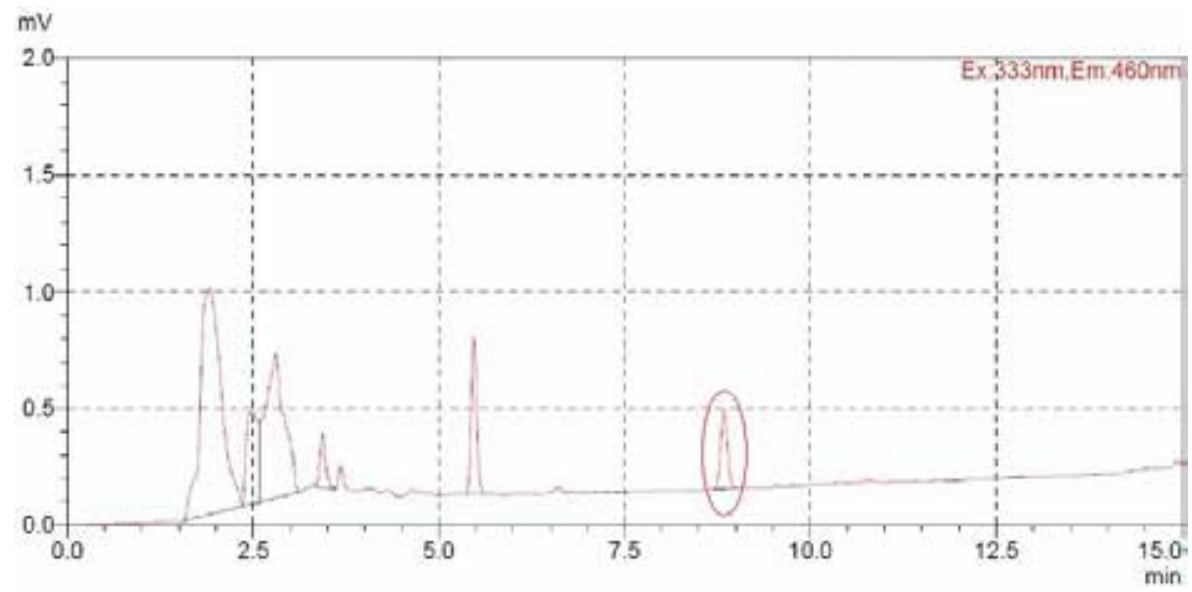

Figura 3. Cromatograma de una de las muestras analizadas

En la figura 4 se puede apreciar un pico (encerrado en círculo) muy cercano al de la OTA. Este pico puede ser un compuesto similar a la OTA, como por ejemplo la ocratoxina B (OTB), ocratoxina C (OTC) o algún otro compuesto producto del metabolismo de la OTA. Di Stefano et al. ${ }^{11}$ encontraron que diversas especies de hongos del tipo Aspergillus producen OTA y OTB de forma paralela, donde la proporción de las toxinas presentes dependían de las 
condiciones del cultivo, aunque el contenido de OTA mostró ser generalmente mayor. Se ha demostrado también que la OTA puede convertirse en OTB y viceversa. De forma similar, se ha demostrado que la ocratoxina $\mathrm{C}$ puede convertirse en OTA en condiciones naturales ${ }^{12}$.

La contaminación por OTA puede ocurrir en diversas etapas del proceso productivo de cacao y derivados, siendo la etapa más proclive la del fermentado, aumentando la proliferación de hongos productores de OTA debido al aumento de temperatura y humedad, suficientes para la aparición de dichos microorganismos; de igual forma, durante la fermentación los granos de cacao se hinchan de líquido, de tal manera que dentro de los granos se crea el ambiente proclive para la generación de estos organismos. La presencia de los hongos productores de OTA se ve disminuida durante la etapa de secado, siempre cuando esta se realice bajos las condiciones adecuadas. Un secado es adecuado cuando disminuye la humedad hasta un $7 \%$ y se realiza bajo sol, ya que la radiación directa elimina buena parte de hongos. Sin embargo, la presencia de hongos productores de OTA puede volver a incrementar durante el almacenado; por ejemplo, cuando la humedad y la temperatura de la zona de almacenamiento no se controlan, esta proliferación se ve aumentada por el hecho de que los granos suelen almacenarse en sacos de yute, lo que genera el ambiente cerrado y oscuro necesario para la formación de los hongos productores de $\mathrm{OTA}^{8,13}$.

A través del análisis micotoxigénico se evidencio que las semillas del cacao, sometidas a esta investigación, presentan hongos miceliales de los géneros Penicillium sp y Fusarium sp, así como la presencia de levaduras en las etapas de secado y fermentado, existiendo una mayor presencia de levaduras en la etapa fermentado $(4,6 \times 104 \mathrm{ufc} / \mathrm{mL})$ por las condiciones mismas del proceso que demanda mayor humedad. En la etapa de almacenado no se evidenciaron presencia de hongos miceliaes ni de levaduras, lo cual es un indicador de las buenas condiciones de almacenamiento.

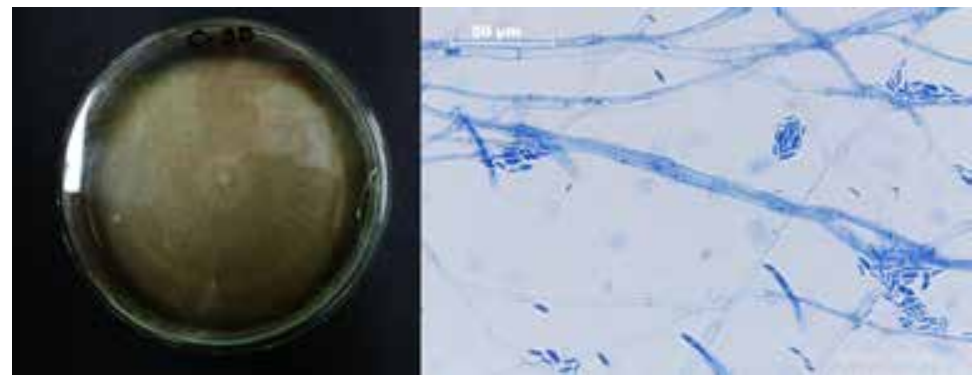

Figura 4. Fusarium sp. en medio APD y microcultivo a los seis días a 400x, respectivamente. 


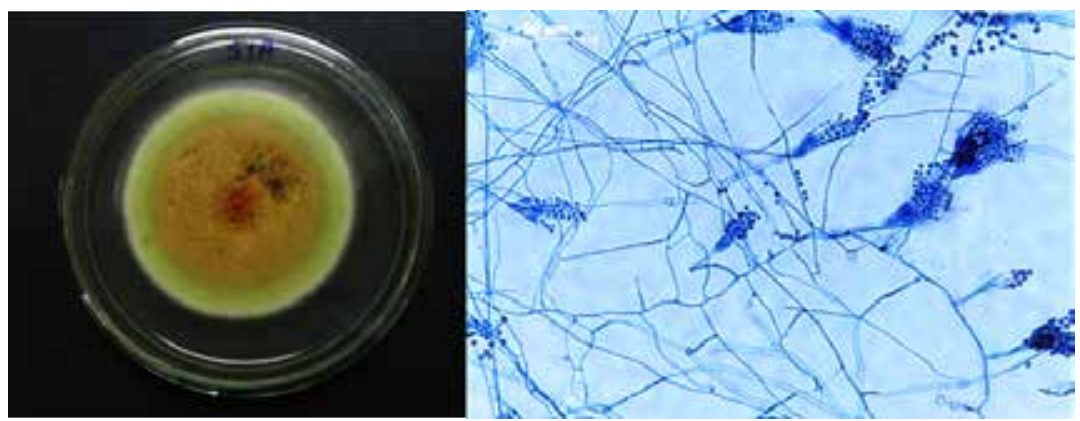

Figura 5. Penicillium sp. en medio APD y microcultivo a los tres días a 400x, respectivamente.

El hecho que las muestras no hayan presentado presencia de OTA demuestra que las prácticas de almacenado, llevadas en el centro de acopio de Pisana-Tingomaría, son efectivas para proteger el cacao de la contaminación por OTA, pero no de la presencia de hongos potenciales productores de micotoxinas, corroborado en el estudio.

Con el fin de profundizar más en los estudios de análisis y detección de OTA en cacao, se recomienda evaluar una mayor cantidad de solventes de extracción y las condiciones en las cuales se realiza dicho proceso, de igual forma se deben evaluar otras columnas de inmunoafinidad con el fin de compararlas con la ya estudiada, además; enfatizar el desarrollo del análisis micotoxigénico que evidencia la carga fúngica de las muestras. En lo que respecta al estudio de la presencia de OTA en granos de cacao peruano, se debe aumentar el número de zonas evaluadas, así como las temporadas de cosecha.

\section{CONCLUSIONES}

El método de extracción de OTA utilizado demostró ser altamente eficaz en la extracción de OTA en granos de cacao para los tres niveles evaluados $(0,5 ; 7,5$ y $15 \mu \mathrm{g} / \mathrm{kg})$. La concentración de ocratoxina A, en las muestras estudiadas de granos de cacao, fueron menores al límite de detección, y menores al límite máximo permitido $(0,5 \mu \mathrm{g} / \mathrm{kg})$. En el análisis micotoxigénico se evidenció la presencia de hongos del género Fusarium, Penicillium, levaduras y la no correspondencia entre ausencia de OTA y la presencia de hongos.

\section{AGRADECIMIENTOS}

Al Vicerrectorado de Investigación y Posgrado de la Universidad Nacional Mayor de San Marcos por el financiamiento del estudio Código: A17040321-2017. 


\section{REFERENCIAS BIBLIOGRÁFICAS}

1. Malir F, Ostry V, Pfohl-Leszkowicz A, Novotna E. Ochratoxin A: Developmental and Reproductive Toxicity-An Overview. Birth Defects Res B Dev Reprod Toxicol. 2013; 98(6): 493-502.

2. SugitakonishI Y, Tanaka T, Nakajima M, Fujita K, Norizuki H, Mochizuki N et.al. The comparison of two clean-up procedures, multifunctional column and immunoaffinity column, for HPLC determination of ochratoxin A in cereals, raisins and green coffee beans. Talanta. 2006; 69(3): 650-655.

3. Afsah-Hejri L, Jinap S, Mirhosseini H. Ochratoxin A quantification: Newly developed HPLC conditions. Food Control. 2012; 23(1): 113-119.

4. Copetti MV, Iamanaka BT, Pitt JI, Taniwaki MH. Fungi and mycotoxins in cocoa: From farm to chocolate. Int J Food Microbiol. 2014; 178: 13-20.

5. Tafuri A, Ferracane R, Ritieni A. Ochratoxin A in Italian marketed cocoa products. Food Chem. 2014; 88(4); 487-494.

6. Mishra RK, Catanante G, Hayat A, Marty JL. Evaluation of extraction methods for ochratoxin A detection in cocoa beans employing HPLC. Food Addit Contam Part A Chem Anal Control Expo Risk Assess. 2016; 33(3): 500-508. doi: 10.1080/19440049.2015.1133933

7. Ramos N, Castro A, Juárez J, Acha O, Rodríguez N, Blancas J et al. Evaluación de ocratoxina A en Theobroma cacao L. "cacao blanco" durante el proceso de cosecha, fermentado, secado y almacenado. Rev Soc Quím Perú. 2017; 82(4): 431-439.

8. Chire G, Valdivia R, Ureña M. Ocratoxina A en cacao y derivados. Medidas preventivas. Ciencia e Investigación. 2014; 17(1): 9-15.

9. Neogen. Neocolumn for Ochratoxin WB quantitative test. [Internet] 2014. [citado 12 enero 2018]. Disponible en: https://foodsafety.neogen.com/en/neocolumn-ochratoxin

10. Hackbart H, Prietto L, Primel E, Garda-Buffon J, Badiale-Furlong E. Simultaneous extraction and detection of ochratoxin A and citrinin in rice. J Braz Chem Soc. 2012; 23(1): 103-109.

11. Di Stefano V, Avellone G, Pitonzo R, Capocchiano V, Mazza A, Cicero N, et al. Natural co-occurrence of ochratoxin A, ochratoxin B and aflatoxins in Sicilian red wines. Food Addit Contam Part A Chem Anal Control Expo Risk Assess. 2015; 32(8): 1343-1351.

12. Heussner A, Bingle L. Comparative Ochratoxin Toxicity: A Review of the Available Data. Toxins. 2015; 7(10): 4253-4282.

13. Copetti M, Iamanaka B, Mororó R, Pereira J, Frisvad J, Taniwaki M, The effect of cocoa fermentation and weak organic acids on growth and ochratoxin A production by Aspergillus species. Int J Food Microbiol. 2012; 155(3): 158-164.

14. Rodríguez G. Caracterización morfológica de hongos endófitos asociados a cacao nativo y su capacidad antagónica para el control de Moniliophthora perniciosa. [tesis para optar al título profesional de ingeniero agrónomo]. Lima: Universidad Nacional de San Martín, Facultad de Ciencias Agrarias; 2011.

15. Arbelaez G. Algunos aspectos de los hongos del género Fusarium y de la especie Fusarium oxysporum. Rev Agron Colomb. 2000; 17: 11-22. 